\title{
GLOBAL AND BLOW-UP SOLUTIONS FOR THE NONLOCAL $p$-LAPLACIAN EVOLUTION EQUATION WITH WEIGHTED NONLINEAR NONLOCAL BOUNDARY CONDITION
}

\author{
ZHONG BO FANG AND JIANYUN ZHANG \\ Communicated by Stig-Olof Londen
}

\begin{abstract}
In this paper, we investigate global existence and blow-up properties of nonnegative solutions to a nonlocal $p$-Laplacian evolution equation with weighted nonlinear nonlocal boundary condition. By using the method of upper and lower solutions, we consider some effects of weight function and nonlinear exponent on the global and blow-up solutions. In addition, we show the blow-up rate estimate, blow-up profile and blow-up set for linear diffusion case.
\end{abstract}

1. Introduction. In this paper, we consider a $p$-Laplacian evolution equation with a nonlocal source term

$$
u_{t}-\operatorname{div}\left(|\nabla u|^{p-2} \nabla u\right)=u^{m} \int_{\Omega} u^{n}(y, t) d y, \quad(x, t) \in \Omega \times(0, T),
$$

subject to weighted nonlinear nonlocal boundary and initial conditions

$$
\begin{aligned}
& u(x, t)=\int_{\Omega} \varphi(x, y) u^{l}(y, t) d y, \quad(x, t) \in \partial \Omega \times(0, T), \\
& u(x, 0)=u_{0}(x), \quad x \in \Omega,
\end{aligned}
$$

where $p>1, m \geq 0, n>0, l>0$ and $\Omega \subset R^{N}(N \geq 1)$ is a bounded domain with smooth boundary. Here, $\varphi(x, y) \not \equiv 0$ is a nonnegative

2010 AMS Mathematics subject classification. Primary 35K65, 35B33, 35B40.

Keywords and phrases. p-Laplacian evolution equation, nonlocal boundary condition, weight function, global existence, blow-up.

This work was supported by the Natural Science Foundation of Shandong Province of China (No. ZR2012AM018) and the Fundamental Research Funds for the Central Universities (No. 201362032).

The first author is the corresponding author.

Received by the editors on January 5, 2013 and in revised form on May 30, 2013. DOI:10.1216/JIE-2014-26-2-171 
continuous function on $\partial \Omega \times \bar{\Omega}$ and $\int_{\Omega} \varphi(x, y) d y>0$ on $\partial \Omega$, and the initial data $u_{0}(x) \in C(\bar{\Omega}) \cap W_{0}^{1, p}(\Omega)$ is a nonnegative function satisfying the compatibility condition.

A nonlinear parabolic equation like (1.1) appears to describe some physical phenomena such as the diffusion of temperature of some combustible substance or the concentration of non-Newton flow in porous medium, see $[\mathbf{1}, \mathbf{2}]$. In the latter case, the quantity $p$ characterizes the medium, i.e., media with $p>2$ and $p<2$ are called dilatant fluids and pseudoplastics, respectively, and if $p=2$, it is said to be Newtonian fluids. In particular, equation (1.1) is a possible model for the diffusion system of some biological species with human-controlled distribution in which case the function $u(x, t)$ represents the density of the species at position $x$ and time $t, \operatorname{div}\left(|\nabla u|^{p-2} \nabla u\right)$ portrays the mutation which can be viewed as an extension of the characteristics, and $u^{m} \int_{\Omega} u^{n} d x$ denotes the human-controlled distribution. The nonlocal term indicates that evolution of species at a point of space does not depend only on nearby density but also on the total amount of species due to the effects of spatial inhomogeneity, see $[\mathbf{1}, \mathbf{3}, \mathbf{1 0}]$.

In the last few decades, there have been many researchers dealing with blow-up solutions to the initial boundary value problems with nonlocal terms or without nonlocal terms in boundary conditions, refer to $[\mathbf{7}, \mathbf{1 6}, \mathbf{2 1}]$ and the references therein. Recently, great attention has been paid to the study of the initial boundary value problem with weighted linear nonlocal boundary conditions. Friedman $[\mathbf{9}]$ and Pao [18] considered a initial boundary value problem of linear and semilinear parabolic equation, respectively. They studied the asymptotic behavior of the solutions and found some effects of weight function on the global and blow-up solutions. For other studies on such problems, one can refer to $[\mathbf{1 4}, \mathbf{1 9}, \mathbf{2 0}]$. For the initial boundary value problem with nonlocal terms and weighted linear nonlocal terms in the boundary condition, i.e., $l=1$ in (1.2), Liu et al. [15] studied the competitive relationship between nonlocal source and absorption terms in a semilinear parabolic equation and found some effects of weight function on the solutions by using Green's function. Wang et al. [22] studied an initial boundary value problem of a porous medium equation with terms of power form. They obtained global existence and blow-up properties of the solutions and blow-up rate estimates by virtue of the method of upper and lower solutions. Cui et al. [4] discussed global existence and 
blow-up properties of the solutions to a slow diffusion porous medium equation with nonlocal source term and linear nonlocal boundary condition. For the coupled system and initial boundary problem of the nonlocal $p$-Laplacian evolution equation with homogeneous Dirichlet boundary condition, refer to $[\mathbf{1 2}, \mathbf{2 5}]$ and the references therein for details.

Recently, Gladkov et al. [11] considered a semi-linear parabolic equation with weighted local source term and weighted nonlinear nonlocal Dirichlet boundary condition

$$
u(x, t)=\int_{\Omega} \varphi(x, y, t) u^{l}(y, t) d y, \quad(x, t) \in \partial \Omega \times(0, \infty),
$$

where $l>0$ and $\varphi(x, y, t)$ is nonnegative. They found effects of weight function and nonlinear exponent on the global and blow-up solutions by the comparison principle and suitable upper and lower solutions. Wang et al. [23] studied a semi-linear parabolic equation with nonlocal source and interior absorption terms and weighted nonlinear nonlocal boundary condition (1.2). They found effects of weight function on the solutions by using Green's function and the technique of upper and lower solutions. Indeed, there are some important phenomena which can be formulated as parabolic equations coupled with weighted nonlocal boundary conditions in the mathematical model, such as thermoelasticity theory. In this case, the solution $u(x, t)$ describes entropy per volume of the materia1 (cf. $[\mathbf{5}, \mathbf{6}]$ ).

Motivated by the above-mentioned works, we investigate global existence and the blow-up behavior of solutions to a nonlocal quasi-linear parabolic equation (1.1) with weighted nonlinear nonlocal boundary condition and reveal some influence of weight function and nonlinear exponent on the solutions. We could obtain some results from the investigation on equation (1.1) with $p>1$ by establishing a modified comparison principle and constructing some suitable upper and lower solutions including a self-similar lower solution, as well as the techniques of the eigenfunction argument and the ordinary differential equation. Specifically, in this paper we treat the nonlocal nonlinear Hölder (non-Lipschitz) cases ( $m$ or $n \in(0,1)$ ), as well as the Lipschitz cases $(m, n \geq 1)$. In fact, obvious differences exist among the situations of slow diffusion $(p>2)$, fast diffusion $(1<p<2)$ and linear diffusion $(p=2)$. For example, there is a finite propagation speed in the 
slow diffusion situation, whereas an infinite propagation speed exists in the fast diffusion situation. We also consider blow-up rate estimate, blow-up profile and blow-up set when $p=2$, in which one can see that our blow-up rate is similar to the one of the equation with local source term. The detailed results are stated below.

Theorem 1.1. Assume that $\int_{\Omega} \varphi(x, y) d y \geq 1$ for $x \in \partial \Omega$ and $l \geq 1$. If $p>1$ and $m+n>1$, then the solution of problems (1.1)-(1.3) blows up in finite time for sufficiently large initial data.

Theorem 1.2. Assume that $\int_{\Omega} \varphi(x, y) d y \leq 1$ for $x \in \partial \Omega$ and $l \leq 1$. If $p>1$ and $m+n \leq 1$, then the solution of problems (1.1)-(1.3) globally exists for small initial data.

Theorem 1.3. Assume that $\int_{\Omega} \varphi(x, y) d y \leq \rho<1$ for $x \in \partial \Omega$ and $l \leq 1$.

(1) If $p>m+n+1$, then the solution of problems (1.1)-(1.3) globally exists for arbitrary initial data.

(2) If $p=m+n+1$, then the solution of problems (1.1)-(1.3) globally exists for small $|\Omega|$.

(3) If $1<p<m+n+1$, then the solution of problems (1.1)-(1.3) globally exists for small initial data.

Remark 1.4. The results of Theorem 1.3 hold for all $p>1$, and we have the following optimal results:

(1) Let $n=p-1$ and $m=0$. If $u_{0}$ satisfies the inequality

$$
\operatorname{div}\left(\left|\nabla u_{0}\right|^{p-2} \nabla u_{0}\right)+u_{0}^{m} \int_{\Omega} u_{0}^{n} d y>0, \quad x \in \Omega,
$$

and $\Omega$ contains a sufficiently large ball $B_{R}(0)$, then the solution of problems (1.1)-(1.3) blows up in finite time.

(2) If $m=p$ and $n=0$, then the solution of problems (1.1)-(1.3) blows up in finite time for sufficiently large initial data.

Theorem 1.5. Assume that $\int_{\Omega} \varphi(x, y) d y \leq 1$ for $x \in \partial \Omega$ and $l \geq 1$. If $1<p \leq 2, m+n>1, m \geq 1$ and $\Omega$ is sufficient small, then the solution of problems (1.1)-(1.3) globally exists for small initial data. 
Theorem 1.6. Assume that $\int_{\Omega} \varphi(x, y) d y \leq 1$ for $x \in \partial \Omega$ and $l \geq 1$. If $1<p<m+n+1$ and $u_{0}(x) \leq \sigma \phi(x)$, where $\phi(x)$ is a function defined in (3.1)-(3.2), then the solution of problems (1.1)-(1.3) globally exists.

Theorem 1.7. Assume that the weight function $\varphi(x, y)>0$ and $l>0$. If $2<p<m+n+1$ and $m+n>1$, then the solution of problems (1.1)-(1.3) blows up in finite time for sufficiently large initial data.

Remark 1.8. The result of Theorem 1.7 is still true for $p=2$ and $l>1$ (cf. [11]).

Remark 1.9. The above results still hold for the fast diffusion case $(1<p<2)$. To the best of our knowledge, no researcher has investigated global and blow-up solutions to nonlocal equations with weighted nonlinear nonlocal boundary condition.

In order to find a blow-up rate estimate for the linear diffusion case $(p=2)$, we assume that the initial data satisfies the following two conditions:

(C1) $\Delta u_{0}(x)+u_{0}^{m} \int_{\Omega} u_{0}^{n} d y>0, x \in \Omega$,

(C2) There exists a constant $\delta>0$ such that $\Delta u_{0}(x)+u_{0}^{m} \int_{\Omega} u_{0}^{n} d y-$ $\delta u_{0}^{m+n} \geq 0$.

Theorem 1.10. Assume that $\int_{\Omega} \varphi(x, y) d y \leq 1$ for $x \in \partial \Omega, l \leq 1$, $p=2$ and $m+n>1$. If the conditions (C1)-(C2) hold, then the blow-up solution of problems (1.1)-(1.3) satisfies

$$
c(T-t)^{-1 /(m+n-1)} \leq u(x, t) \leq C(T-t)^{-1 /(m+n-1)},
$$

where $c=[|\Omega|(m+n-1)]^{-1 /(m+n-1)}$ and $C=[\delta(m+n-1)]^{-1 /(m+n-1)}$.

Finally, we obtain the blow-up profile for the linear diffusion case.

Theorem 1.11. Assume that $\int_{\Omega} \varphi(x, y) d y<1$ for $x \in \partial \Omega, l \leq 1$, $p=2, m<1$ and $m+n>1$. If $\Delta u_{0} \leq 0$ in $\bar{\Omega}$, we then have

$$
\lim _{t \rightarrow T}(T-t)^{-1 /(m+n-1)} u(x, t)=M_{1}
$$


where

$$
M_{1}=\left(|\Omega| \frac{m+n-1}{1-m}\right)^{-1 /(m+n-1)}(1-m)^{-1 /(m+n-1)} .
$$

The rest of our paper is organized as follows. In Section 2, with the definition of weak upper and lower solutions, we give a modified comparison principle of problems (1.1)-(1.3), which is an important tool for our research. The proofs of results on the global existence and blow-up properties of solutions are given in Section 3. In Section 4, we give the blow-up rate estimate, the blow-up profile and the blow-up set for $p=2$.

2. Comparison principle. Since equation (1.1) is degenerate when $p>2$ and singular when $1<p<2$, there is no classical solution for $p>1$ in general. Hence, it is reasonable to find a weak solution of problem (1.1)-(1.3). To this end, we first give the following definition of nonnegative weak solution of problems (1.1)-(1.3).

Definition 2.1. If a nonnegative function $u(x, t)$ satisfies the following conditions:

$$
\begin{gathered}
\text { (i) } u \in C\left(0, T ; L^{\infty}(\Omega)\right) \bigcap L^{p}\left(0, T ; W_{0}^{1, p}(\Omega)\right), \\
u_{t} \in L^{2}\left(0, T ; L^{2}(\Omega)\right), u(x, 0)=u_{0}(x), \\
\text { (ii) } \iint_{Q_{T}} u_{t} \phi d x d t+\iint_{Q_{T}}|\nabla u|^{p-2} \nabla u \cdot \nabla \phi d x d t \\
=\iint_{Q_{T}} \phi\left(u^{m} \int_{\Omega} u^{n}(y, t) d y\right) d x d t,
\end{gathered}
$$

where $\phi \in L^{1}\left(0, T ; W^{1,2}(\Omega)\right) \bigcap L^{2}\left(Q_{T}\right), \phi(x, t)=0$ on $\partial \Omega$ and $Q_{T}=$ $\Omega \times(0, T)$

$$
\text { (iii) } u(x, t)=\int_{\Omega} \varphi(x, y) u^{l}(y, t) d y, \quad x \in \partial \Omega,
$$

then $u(x, t)$ is called a weak solution of problems (1.1)-(1.3). 
A lower solution $\underline{u}(x, t)$ and an upper solution $\bar{u}(x, t)$ of problems (1.1)-(1.3) can be similarly defined by replacing the equality sign in (2.1) with inequality signs $\leq$ and $\geq$, respectively.

Lemma 2.2. Let $\xi, \bar{\xi} \in R^{N}$. There exists a positive constant $c=$ $c(N, p)$ such that

(i) if $p>2$, then $\left(|\bar{\xi}|^{p-2} \bar{\xi}-|\xi|^{p-2} \xi\right) \cdot(\bar{\xi}-\xi) \geq c|\bar{\xi}-\xi|^{p}$,

(ii) if $1<p \leq 2$, then $\left(|\bar{\xi}|^{p-2} \bar{\xi}-|\xi|^{p-2} \xi\right) \cdot(\bar{\xi}-\xi) \geq c \frac{|\overline{\xi-\xi}|^{2}}{(|\bar{\xi}|+|\xi|)^{2}}$, $(|\bar{\xi}|+|\xi| \neq 0)$.

Remark 2.3. Lemma 2.2 can be shown in a similar manner as the one used in [8, Lemma 4.10].

We prove the following modified comparison principle by establishing the suitable test function and using Lemma 2.2 and Gronwall's inequality.

Proposition 2.4. (Comparison principle). Suppose that $\underline{u}(x, t)$ and $\bar{u}(x, t)$ is a lower solution and upper solution of problems (1.1)-(1.3), respectively, with $\underline{u}(x, 0) \geq 0, \bar{u}(x, 0) \geq \varepsilon>0$, and $\underline{u}(x, 0) \leq \bar{u}(x, 0)$, where $\varepsilon$ is any positive constant. We then have $\underline{u}(x, t) \leq \bar{u}(x, t)$ in $\bar{\Omega} \times(0, T)$.

Proof. For $x \in \Omega$, we have the inequality

$$
\begin{array}{r}
\iint_{Q_{T}}\left(\underline{u}_{t}-\bar{u}_{t}\right) \phi d x d t+\iint_{Q_{T}}\left(|\nabla \underline{u}|^{p-2} \nabla \underline{u}-|\nabla \bar{u}|^{p-2} \nabla \bar{u}\right) \cdot \nabla \phi d x d t \\
\leq \iint_{Q_{T}} \phi\left[\underline{u}^{m} \int_{\Omega} \underline{u}^{n}(y, t) d y-\bar{u}^{m} \int_{\Omega} \bar{u}^{n}(y, t) d y\right] d x d t
\end{array}
$$

since $\underline{u}$ and $\bar{u}$ are lower and upper solutions of problems (1.1)-(1.3), respectively. Choosing a test function $\phi=(\underline{u}-\bar{u})_{+}=\max \{\underline{u}-\bar{u}, 0\}$, 
we get

$$
\begin{aligned}
& \iint_{Q_{T}}(\underline{u}-\bar{u})_{t}(\underline{u}-\bar{u})_{+} d x d t \\
& +\iint_{Q_{T}}\left(|\nabla \underline{u}|^{p-2} \nabla \underline{u}-|\nabla \bar{u}|^{p-2} \nabla \bar{u}\right) \cdot \nabla(\underline{u}-\bar{u})_{+} d x d t \\
& \quad \leq \iint_{Q_{T}}(\underline{u}-\bar{u})_{+}\left[\underline{u}^{m} \int_{\Omega} \underline{u}^{n}(y, t) d y-\bar{u}^{m} \int_{\Omega} \bar{u}^{n}(y, t) d y\right] d x d t .
\end{aligned}
$$

By Lemma 2.2, we deduce that $\left(|\nabla \underline{u}|^{p-2} \nabla \underline{u}-|\nabla \bar{u}|^{p-2} \nabla \bar{u}\right) \cdot \nabla(\underline{u}-\bar{u})_{+}$ $\geq 0$ for all $p>1$, and so

$$
\begin{aligned}
& \iint_{Q_{T}}(\underline{u}-\bar{u})_{t}(\underline{u}-\bar{u})_{+} d x d t \\
& \leq \iint_{Q_{T}}(\underline{u}-\bar{u})_{+}\left[\underline{u}^{m} \int_{\Omega} \underline{u}^{n}(y, t) d y-\bar{u}^{m} \int_{\Omega} \bar{u}^{n}(y, t) d y\right] d x d t \\
& =\iint_{Q_{T}}\left[\left(\underline{u}^{m}-\bar{u}^{m}\right) \int_{\Omega} \underline{u}^{n}(y, t) d y\right](\underline{u}-\bar{u})_{+} d x d t \\
& \quad+\iint_{Q_{T}}(\underline{u}-\bar{u})_{+} \bar{u}^{m} \int_{\Omega}\left(\underline{u}^{n}-\bar{u}^{n}\right)(y, t) d y d x d t \\
& \leq \iint_{Q_{T}} \Phi_{1}(x, t)(\underline{u}-\bar{u})_{+}^{2}\left(\int_{\Omega} \underline{u}^{n}(y, t) d y\right) d x d t \\
& \quad+|\Omega| \iint_{Q_{T}} \Phi_{2}(x, t)(\underline{u}-\bar{u})_{+}^{2} \bar{u}^{m} d x d t,
\end{aligned}
$$

where

$$
\begin{aligned}
& \Phi_{1}(x, t)=m \int_{0}^{1}(\theta \underline{u}+(1-\theta) \bar{u})^{m-1} d \theta \\
& \Phi_{2}(x, t)=n \int_{0}^{1}(\theta \underline{u}+(1-\theta) \bar{u})^{n-1} d \theta .
\end{aligned}
$$

For $m, n \geq 1$, the functions $\Phi_{i}(i=1,2)$ are bounded and, if $0 \leq m<1$ or $0<n<1$, we have $\Phi_{1} \leq \varepsilon^{m-1}$ and $\Phi_{2} \leq \varepsilon^{n-1}$, since $\underline{u}(x, 0) \geq 0$ and $\bar{u}(x, 0) \geq \varepsilon>0$. Furthermore, $\underline{u}(x, t)$ and $\bar{u}(x, t)$ are bounded functions, and hence, we have the inequality

$$
\iint_{Q_{T}}(\underline{u}-\bar{u})_{t}(\underline{u}-\bar{u})_{+} d x d t \leq C \iint_{Q_{T}}(\underline{u}-\bar{u})_{+}^{2} d x d t
$$


for some constant $C>0$.

On the other hand, it follows from $\underline{u}(x, 0) \leq \bar{u}(x, 0)$ that

$$
\begin{aligned}
\int_{\Omega}(\underline{u}-\bar{u})_{+}^{2} d x & \leq \int_{\Omega}(\underline{u}-\bar{u})_{+}^{2}-\int_{\Omega}(\underline{u}(x, 0)-\bar{u}(x, 0))_{+}(\underline{u}-\bar{u})_{+} d x \\
& \leq C \iint_{Q_{T}}(\underline{u}-\bar{u})_{+}^{2} d x d t .
\end{aligned}
$$

By Gronwall's inequality, we can deduce $(\underline{u}-\bar{u})_{+}=0$, and hence, we get $\underline{u}(x, t) \leq \bar{u}(x, t)$ in $\Omega \times(0, T)$.

For $x \in \partial \Omega$, we have

$$
\underline{u}(x, t)-\bar{u}(x, t) \leq \int_{\Omega} \varphi(x, y)\left(\underline{u}^{l}(y, t)-\bar{u}^{l}(y, t)\right) d y .
$$

Since

$$
\begin{aligned}
\int_{\Omega} \varphi(x, y)\left(\underline{u}^{l}(y, t)-\bar{u}^{l}(y, t)\right) d y & =\int_{\Omega_{1}} \varphi(x, y)\left(\underline{u}^{l}(y, t)-\bar{u}^{l}(y, t)\right) d y \\
& +\int_{\Omega_{2}} \varphi(x, y)\left(\underline{u}^{l}(y, t)-\bar{u}^{l}(y, t)\right) d y,
\end{aligned}
$$

where

$$
\Omega_{1}=\{y \in \Omega: \underline{u}(y, t) \leq \bar{u}(y, t)\}, \quad \Omega_{2}=\{y \in \Omega: \underline{u}(y, t)>\bar{u}(y, t)\},
$$
$\varphi(x, y)$ is continuous, $\underline{u}(x, 0) \geq 0$ and $\bar{u}(x, 0) \geq \varepsilon>0$, we can arrive at

$$
\begin{aligned}
\int_{\Omega} \varphi(x, y)\left(\underline{u}^{l}(y, t)-\bar{u}^{l}(y, t)\right) d y & =\int_{\Omega} \varphi(x, y) \Phi_{3}(y, t)(\underline{u}-\bar{u})_{+} d y \\
& \leq C \int_{\Omega}(\underline{u}-\bar{u})_{+} d y
\end{aligned}
$$

where

$$
\Phi_{3}(x, t)=l \int_{0}^{1}(\theta \underline{u}+(1-\theta) \bar{u})^{l-1} d \theta .
$$

In the case of $\left.\int_{\Omega}(\underline{u}-\bar{u})_{+}\right) d y=0$ in $\Omega$, it can easily be seen that

$$
\underline{u}-\bar{u} \leq \int_{\Omega} \varphi(x, y)\left(\underline{u}^{l}(y, t)-\bar{u}^{l}(y, t)\right) d y \leq 0,
$$

and so $\underline{u}(x, t) \leq \bar{u}(x, t)$ on $\partial \Omega \times(0, T)$. Therefore, we obtain $\underline{u}(x, t) \leq$ $\bar{u}(x, t)$ in $\bar{\Omega} \times(0, T)$. 
Remark 2.5. The existence of local nonnegative solutions in time to problems (1.1)-(1.3) can be obtained by using a fixed point theorem (cf. [24]) or a parabolic regular theory to get a suitable estimate in a standard limiting process, see $[\mathbf{1 2}, \mathbf{2 7}]$. By Proposition 2.4, we get the uniqueness of solution to the problem. The proof is more or less standard, and so it is omitted here.

3. Global existence and blow-up of solutions. Comparing problems (1.1)-(1.3) with initial boundary problems subject to general homogeneous Dirichlet boundary condition, one can see that the existence of the weight function and the nonlinear exponent in the boundary condition has a great influence on the existence of global and non-global solutions.

Proof of Theorem 1.1. Consider the following initial value problem of an ordinary differential equation:

$$
\begin{aligned}
& V^{\prime}(t)=|\Omega| V^{m+n}, \\
& V(0)=V_{0} .
\end{aligned}
$$

It is easy to see that solutions of the above problem blow up in finite time for arbitrary initial data, provided that $m+n>1$.

For $1<V_{0}<\min _{\Omega} u_{0}(x)$, we obtain

$$
\begin{aligned}
V(t) & \leq V^{l}(t) \\
& \leq \int_{\Omega} \varphi(x, y) d y V^{l}(t) \\
& =\int_{\Omega} V^{l}(t) \varphi(x, y) d y \quad \text { for } x \in \partial \Omega,
\end{aligned}
$$

since $\int_{\Omega} \varphi(x, y) d y \geq 1$ and $l \geq 1$. It is obvious that $V(t)$ is a lower solution of problems (1.1)-(1.3), and hence, the solution $u(x, t)$ of problems (1)-(3) blows up in finite time.

Proof of Theorem 1.2. Let $\bar{u}(x, t)=\alpha e^{\beta t}$, where $\alpha \geq 1$ and $\beta>0$ are constants that will be determined later. We then have

$$
\begin{aligned}
\bar{u}_{t}-\operatorname{div}\left(|\nabla \bar{u}|^{p-2} \nabla \bar{u}\right)-\bar{u}^{m} \int_{\Omega} \bar{u}^{n} d y & =\alpha \beta e^{\beta t}-\alpha^{m+n} e^{m \beta t} \int_{\Omega} e^{n \beta t} d y \\
& \geq \alpha \beta e^{\beta t}-\alpha^{m+n} e^{\beta(m+n) t}|\Omega| .
\end{aligned}
$$


Because of $m+n \leq 1$, we get

$$
\bar{u}_{t}-\operatorname{div}\left(|\nabla \bar{u}|^{p-2} \nabla \bar{u}\right)-\bar{u}^{m} \int_{\Omega} \bar{u}^{n} d y \geq 0
$$

by taking $\alpha=\max _{\bar{\Omega}}\left\{u_{0}, 1\right\}$ and $\beta=|\Omega| \alpha^{m+n-1}$ in the above inequality.

For $x \in \partial \Omega$,

$$
\begin{aligned}
\bar{u}(x, t) & =\alpha e^{\beta t} \geq \alpha e^{\beta t} \int_{\Omega} \varphi(x, y) d y \\
& \geq \int_{\Omega} \varphi(x, y) \alpha^{l} e^{\beta l t} d y \\
& =\int_{\Omega} \varphi(x, y) \bar{u}^{l}(y, t) d y,
\end{aligned}
$$

since $\int_{\Omega} \varphi(x, y) d y \leq 1, \alpha \geq 1$ and $l \leq 1$, which implies that $\bar{u}(x, t)$ is an upper solution of problems (1.1)-(1.3). By Proposition 2.4, one can see that a global solution of problems (1.1)-(1.3) exists.

Proof of Theorem 1.3. Assume that $\psi(x)$ solves the following problem:

$$
\begin{aligned}
-\operatorname{div}\left(|\nabla \psi|^{p-2} \nabla \psi\right)=1, & x \in \Omega, \\
\psi(x) & =0, \quad x \in \partial \Omega .
\end{aligned}
$$

It has been shown that $\psi(x)>0$ in $\Omega$ and $\partial \psi / \partial n<0$, where $n$ is an outward unit normal vector to $\partial \Omega$, see [5]. Let $M=\max _{x \in \bar{\Omega}} \psi(x)<\infty$, and let $\omega(x, t)=k((\rho / 1-\rho)+(\psi(x) / M))$. Here, $k \geq 1$ is a constant that will be determined later.

It can easily be seen that $\omega_{t}=0$ and

$$
-\operatorname{div}\left(|\nabla \omega|^{p-2} \nabla \omega\right)=\left(\frac{k}{M}\right)^{p-1}\left[-\operatorname{div}\left(|\nabla \psi|^{p-2} \nabla \psi\right)\right]=\left(\frac{k}{M}\right)^{p-1} .
$$

Because of $\rho /(1-\rho)+(\psi(x) / M) \leq \rho /(1-\rho)+1=1 /(1-\rho)$, we have

$$
\begin{aligned}
\omega_{t}-\operatorname{div}\left(|\nabla \omega|^{p-2} \nabla \omega\right)-\omega^{m} & \int_{\Omega} \omega^{n}(y, t) d y \\
& \geq\left(\frac{k}{M}\right)^{p-1}-k^{m+n}\left(\frac{1}{1-\rho}\right)^{m+n}|\Omega| .
\end{aligned}
$$


(1) If $p>m+n+1$, we get

$$
\omega_{t}-\operatorname{div}\left(|\nabla \omega|^{p-2} \nabla \omega\right)-\omega^{m} \int_{\Omega} \omega^{n}(y, t) d y \geq 0
$$

by taking $k=\max \left\{\max \left|u_{0}(x)\right|,\left[|\Omega| M^{p-1} 1 /(1-\rho)^{m+n}\right]^{1 /(p-1-m-n)}, 1\right\}$,

(2) If $p=m+n+1$,

$$
\begin{aligned}
\omega_{t}-\operatorname{div}\left(|\nabla \omega|^{p-2} \nabla \omega\right)-\omega^{m} \int_{\Omega} \omega^{n}(y, t) d y & \\
& \geq\left(\frac{k}{M}\right)^{p-1}-k^{p-1}\left(\frac{1}{1-\rho}\right)^{p-1}|\Omega| .
\end{aligned}
$$

Hence,

$$
\left.\omega_{t}-\right] \operatorname{div}\left(|\nabla \omega|^{p-2} \nabla \omega\right)-\omega^{m} \int_{\Omega} \omega^{n}(y, t) d y \geq 0
$$

provided that $|\Omega| \leq(1-\rho / M)^{p-1}$.

(3) If $1<p<m+n+1$, we can have

$$
\omega_{t}-\operatorname{div}\left(|\nabla \omega|^{p-2} \nabla \omega\right)-\omega^{m} \int_{\Omega} \omega^{n}(y, t) d y \geq 0
$$

by taking $k$ such that

$$
\max _{\bar{\Omega}}\left\{\max \left|u_{0}(x)\right|, 1\right\} \leq k \leq\left(M^{1-p}(1-\rho)^{m+n}|\Omega|^{-1}\right)^{1 /(m+n+1-p)} .
$$

For $x \in \partial \Omega$, we consider the following two cases.

Case 1. $1 / 2<\int_{\Omega} \varphi(x, y) d y \leq \rho<1$. Since $\rho /(1-\rho)>1, k \geq 1$, and $l \leq 1$, we obtain

$$
\begin{aligned}
\omega(x, t) & =k\left(\frac{\rho}{1-\rho}\right) \\
& =k\left(\frac{\rho}{1-\rho}+1\right) \rho \geq k\left(\frac{\rho}{1-\rho}+\frac{\psi(x)}{M}\right) \int_{\Omega} \varphi(x, y) d y \\
& \geq \int_{\Omega} k^{l}\left(\frac{\rho}{1-\rho}+\frac{\psi(x)}{M}\right)^{l} \varphi(x, y) d y \\
& =\int_{\Omega} \omega^{l} \varphi(x, y) d y .
\end{aligned}
$$


Case 2. $\int_{\Omega} \varphi(x, y) d y \leq 1 / 2$ (in this case, set $\rho=1 / 2$ ). We then have

$$
\begin{aligned}
\omega(x, t) & =k \geq 2 k \int_{\Omega} \varphi(x, y) d y \\
& \geq \int_{\Omega} k \varphi(x, y)\left(1+\frac{\psi(x)}{M}\right) d y \\
& \geq \int_{\Omega} \varphi(x, y) \omega^{l} d y,
\end{aligned}
$$

provided that $k \geq 1$ and $l \leq 1$. The proof is completed.

Proof of Theorem 1.5. Assume that $\psi(x)$ solves the following problem:

$$
\begin{gathered}
-\operatorname{div}\left(|\nabla \psi|^{p-2} \nabla \psi\right)=\lambda \psi^{p-1}, \quad x \in \Omega, \\
\psi(x)=\int_{\Omega} \varphi(x, y) d y, \quad x \in \partial \Omega,
\end{gathered}
$$

where $\lambda>0$ is a constant. It is known that $0<\psi(x) \leq 1$ in $\bar{\Omega}$, see [6], so $\max _{\bar{\Omega}} \psi(x)=1$. Setting $\omega(x, t)=(A+t)^{-\sigma} \psi(x)$, where $A>0$ is a constant, we obtain

$$
\omega_{t}=-\sigma(A+t)^{-\sigma-1} \psi(x), \quad \nabla \omega=(A+t)^{-\sigma} \nabla \psi(x),
$$

and

$$
\begin{aligned}
\omega_{t}-\operatorname{div}\left(|\nabla \omega|^{p-2}\right. & \nabla \omega)-\omega^{m} \int_{\Omega} \omega^{n}(y, t) d y \\
& =-\sigma(A+t)^{-\sigma-1} \psi(x)+(A+t)^{-\sigma(p-1)} \lambda \psi^{p-1} \\
& -(A+t)^{-\sigma(m+n)} \psi^{m} \int_{\Omega} \psi^{n} d y \\
& =(A+t)^{-\sigma(p-1)} \psi^{p-1}(x)\left[-\sigma(A+t)^{\sigma(p-2)-1} \psi^{2-p}\right. \\
& \left.+\lambda-(A+t)^{\sigma(p-1-m-n)} \psi^{m-p+1} \int_{\Omega} \psi^{n} d y\right] \\
& \geq(A+t)^{-\sigma(p-1)} \psi^{p-1}(x)\left[-\sigma(A+t)^{\sigma(p-2)-1} \psi^{2-p}\right. \\
& \left.+\lambda-(A+t)^{\sigma(p-1-m-n)+1}|\Omega|\right]
\end{aligned}
$$

provided that $m+n>1, m \geq 1$ and $1<p \leq 2$. 
If $A$ is sufficiently large and $|\Omega|$ is small enough, we have

$$
\omega_{t}-\operatorname{div}\left(|\nabla \omega|^{p-2} \nabla \omega\right)-\omega^{m} \int_{\Omega} \omega^{n}(y, t) d y \geq 0
$$

by taking $\sigma>1 /(m+n+1-p)$.

For $x \in \partial \Omega$, set $\omega(x, t)=(A+t)^{-\sigma} \int_{\Omega} \varphi(x, y) d y$. Since $0<\psi(x) \leq 1$ and $l \geq 1$, we get

$$
\begin{aligned}
\omega(x, t) & \geq(A+t)^{-\sigma} \int_{\Omega} \psi(y) \varphi(x, y) d y \\
& \geq \int_{\Omega} \psi^{l}(y)(A+t)^{-\sigma l} \varphi(x, y) d y \\
& =\int_{\Omega} \omega^{l} \varphi(x, y) d y
\end{aligned}
$$

for large enough $A$. Then $\omega(x, t)$ is an upper solution of problems (1.1)-(1.3) if $u_{0} \leq A^{-\sigma} \psi(x)$, which completes the proof.

Proof of Theorem 1.6. Assume that $\phi(x)$ is a solution of the following problem.

$$
\begin{gathered}
-\operatorname{div}\left(|\nabla \phi|^{p-2} \nabla \phi\right)=\lambda, \quad x \in \Omega, \\
\phi(x)=\int_{\Omega} \varphi(x, y) d y, \quad x \in \partial \Omega .
\end{gathered}
$$

Choosing a proper $\lambda$ such that $0<\phi(x)<1$ and setting

$$
v(x, t)=\sigma \phi(x),
$$

where

$$
0<\sigma<\min \left\{1,\left(\frac{\phi^{m} \int_{\Omega} \phi^{n} d y}{\lambda}\right)^{-1 /(m+n+1-p)}\right\},
$$

we have

$$
\begin{aligned}
v_{t}-\operatorname{div}\left(|\nabla v|^{p-2} \nabla v\right) & =\sigma^{p-1}\left(-\operatorname{div}\left(|\nabla \phi|^{p-2} \nabla \phi\right)\right. \\
& =\sigma^{p-1} \lambda>\sigma^{m+n} \phi^{m} \int_{\Omega} \phi^{n} d y .
\end{aligned}
$$


On the other hand, since $l \geq 1$, we get

$$
\begin{aligned}
v(x) & =\sigma \int_{\Omega} \varphi(x, y) d y \geq \int_{\Omega} \sigma \phi(y) \varphi(x, y) d y \\
& \geq \int_{\Omega} \sigma^{l} \phi^{l}(y) \varphi(x, y) d y=\int_{\Omega} v^{l} \varphi(x, y) d y .
\end{aligned}
$$

Hence, $v(x, t)$ is an upper solution of problems (1.1)-(1.3) provided that $u_{0}(x) \leq \sigma \phi(x)$, which means that the solution of problems (1.1)-(1.3) globally exist for sufficiently small initial data.

Proof of Theorem 1.7. In order to prove the blow-up result, we need to establish a self-similar blow-up solution. We first assume that $\varphi \in C^{1}(\bar{\Omega}), \varphi(x) \geq 0, \varphi(x)$ is not identically zero, and $\left.\varphi(x)\right|_{\partial \Omega}=0$. Without loss of generality, we assume that $0 \in \Omega$ and $\varphi(0)>0$.

Let $\underline{u}(x, t)=(T-t)^{-\gamma} V(\xi)$, where $V(\xi)=\left(1+(A / 2)-\left(\xi^{2} / 2 A\right)\right)_{+}$, $\xi=|x|(T-t)^{-\mu}, A>1,0<T<1$ and $\gamma, \mu>0$. It is easy to see that

$$
\left.\left.\operatorname{supp} \underline{u}_{+}(\cdot, t)=\overline{B\left(0, R(T-t)^{\mu}\right.}\right) \subset \overline{B\left(0, R T^{\mu}\right.}\right) \subset \Omega
$$

for sufficiently small $T>0$ and $R=\sqrt{A(A+2)}$. Calculating the derivative of $\underline{u}$ with respect to the variable $t$, we obtain

$$
\begin{aligned}
& \underline{u}_{t}=\frac{\gamma V(\xi)}{(T-t)^{\gamma+1}}+\frac{\mu V^{\prime}(\xi)|x|}{(T-t)^{\gamma}(T-t)^{\mu+1}}=\frac{\gamma V(\xi)+\mu \xi V^{\prime}(\xi)}{(T-t)^{\gamma+1}}, \\
& \nabla \underline{u}=\nabla\left[(T-t)^{-\gamma}\left(1+\frac{A}{2}\right)\right]=(T-t)^{-\gamma}\left(-\frac{\xi}{A}\right) \nabla \xi \\
&=(T-t)^{-\gamma-\mu}\left(-\frac{|x|(T-t)^{-\mu}}{A}\right) \nabla(|x|), \\
& \triangle \underline{u}=\frac{N}{A(T-t)^{\gamma+2 \mu}}, \\
& \operatorname{div}\left(|\nabla \underline{u}|^{p-2} \nabla \underline{u}\right)=|\nabla \underline{u}|^{p-2} \triangle \underline{u}+(p-2)|\nabla \underline{u}|^{p-4}(\nabla \underline{u})^{\prime}\left(H_{x}(\underline{u})\right) \cdot \nabla \underline{u} \\
&=|\nabla \underline{u}|^{p-2} \triangle \underline{u}+(p-2)|\nabla \underline{u}|^{p-4} \sum_{j=1}^{N} \sum_{i=1}^{N} \frac{\partial \underline{u}}{\partial x_{i}} \frac{\partial \underline{u}}{\partial x_{i} \partial x_{j}} \frac{\partial \underline{u}}{\partial x_{j}}
\end{aligned}
$$

where $H_{(x)}(\underline{u})$ is the Hessian matrix of $\underline{u}$. Let $d(\Omega)=\operatorname{diam}(\Omega)$. We 
then have

$$
\begin{aligned}
\left|\operatorname{div}\left(|\nabla \underline{u}|^{p-2} \nabla \underline{u}\right)\right| \leq \frac{N(p-1)(d(\Omega))^{p-2}}{A(T-t)^{(\gamma+2 \mu)(p-1)}} & \\
\int_{\Omega} \underline{u}^{n}(y, t) d y & =\frac{1}{(T-t)^{n \gamma}} \int_{B\left(0, R(T-t)^{\mu}\right)} V^{n}\left(\frac{|x|}{(T-t)^{\mu}}\right) d x \\
& =\frac{M}{(T-t)^{n \gamma-N \mu}},
\end{aligned}
$$

where $M=\int_{B(0, R)} V^{n}(|\xi|) d \xi>0$.

If $0 \leq \xi \leq A$, then $V^{\prime}(\xi) \leq 0$. It can easily be seen that

$$
M \geq \int_{B(0, A)} V^{n}(|\xi|) d \xi \geq\|B(0, A)\| \quad \text { for } R>A
$$

and

$$
\begin{aligned}
\underline{u}_{t}- & \operatorname{div}\left(|\nabla \underline{u}|^{p-2} \nabla \underline{u}\right)-\underline{u} \int_{\Omega} \underline{u}^{n}(y, t) d y \\
\leq & \frac{\gamma V(\xi)+\mu \xi V^{\prime}(\xi)}{(T-t)^{\gamma+1}}+\frac{N(p-1)(d(\Omega))^{p-2}}{A(T-t)^{(\gamma+2 \mu)(p-1)}} \\
& -\frac{V(\xi)}{(T-t)^{m \gamma+n \gamma}} \int_{\Omega} \underline{u}^{n}(x, t) d x \\
\leq & \frac{\gamma(1+2 A)}{(T-t)^{\gamma+1}}+\frac{N(p-1)(d(\Omega))^{p-2}}{A(T-t)^{(\gamma+2 \mu)(p-1)}}-\frac{\|B(0, A)\|}{(T-t)^{m \gamma+n \gamma-N \mu}} .
\end{aligned}
$$

If $\xi \geq A$, we have $V^{\prime}(\xi)=-\xi / A \leq-1$ and $V(\xi) \leq 1$. It follows from (3.2) and $M>0$ that

$$
\begin{aligned}
\underline{u}_{t}-\operatorname{div}\left(|\nabla \underline{u}|^{p-2} \nabla \underline{u}\right)- & \underline{u} \int_{\Omega} \underline{u}^{n}(x, t) d x \\
& \leq \frac{\gamma-\mu A}{(T-t)^{\gamma+1}}+\frac{N(p-1)(d(\Omega))^{p-2}}{A(T-t)^{(\gamma+2 \mu)(p-1)}}
\end{aligned}
$$


Since $1<p<m+n+1$, we can choose constants $\gamma, \mu$ and $A$ such that

$$
\begin{aligned}
\frac{1+N \mu}{m+n-1} & <\gamma<\frac{1-2 \mu(p-1)}{p-2} \\
0 & <\mu<\frac{m+n+1-p}{N(p-2)+2(p-1)(m+n-1)}, \\
A & >\left\{1, \frac{\gamma}{\mu}, \frac{N(p-1)(d(\Omega))^{p-2}}{\mu}\right\}
\end{aligned}
$$

We then obtain

$$
\underline{u}_{t}-\operatorname{div}\left(|\nabla \underline{u}|^{p-2} \nabla \underline{u}\right)-\underline{u} \int_{\Omega} \underline{u}^{n}(y, t) d y \leq 0 \quad \text { in } Q_{T}
$$

for sufficiently small $T>0$ by (3.5) and (3.6).

For $x \in \partial \Omega$, since $\varphi(0)>0$ and $\varphi$ is continuous, it is known that positive constants $\varepsilon$ and $\rho$ exist such that $\varphi \geq \varepsilon$ for $x \in B(0, \rho)$. Furthermore, we have $B\left(0, R T^{\sigma}\right) \subset B(0, \rho) \subset \Omega$, if $T$ is small enough, and so $\underline{u} \leq \int_{\Omega} \varphi(x, y) \underline{u}^{l} d y$ on $\partial \Omega \times(0, T)$. It follows from (3.3) that $\underline{u}(x, 0) \leq K_{0} \varphi(x)$ for sufficiently large $K_{0}$. Therefore, one can see that the solutions to problems (1.1)-(1.3) exist no later than $t=T$, provided that $u_{0} \geq K_{0} \varphi(x)$. This implies that the solution blows up in finite time for large enough initial data.

4. Blow-up rate estimate and blow-up profile. In this section, we give blow-up rate, blow-up profile and blow-up set of the solution to problems (1.1)-(1.3) for the linear diffusion case $(p=2)$.

4.1. Blow-up rate estimate. Let $u(x, t)$ be a blow-up solution of problems (1.1)-(1.3) for the linear diffusion case, and let

$$
U(t)=\max _{x \in \bar{\Omega}} u(x, t) .
$$

Lemma 4.1. If $u_{0}$ satisfies conditions $(\mathrm{C} 1)-(\mathrm{C} 2)$, then a positive constant $c=[(m+n-1)|\Omega|]^{-1 /(m+n-1)}$ exists such that

$$
U(t) \geq c(T-t)^{-1 /(m+n-1)} .
$$


Proof. It is obvious that $U(t)$ is Lipschitz continuous and differentiable almost everywhere. It follows from $\triangle U(t) \leq 0$ that

$$
U^{\prime}(t) \leq U^{m} \int_{\Omega} U^{n} d y \leq|\Omega| U^{m+n} .
$$

We then have

$$
-\left(U^{1-m-n}(t)\right)^{\prime} \leq|\Omega|(m+n-1) .
$$

Integrating the inequality above over $(t, T)$, the desired result follows.

Proof of Theorem 1.10. Let $J=u_{t}-\delta u^{m+n}$. We then have

$$
\begin{aligned}
J_{t} & =u_{t t}-\delta(m+n) u^{m+n-1} u_{t} \\
& =\triangle u_{t}+m u^{m-1} u_{t} \int_{\Omega} u^{n} d x+n u^{m} \int_{\Omega} u^{n-1} u_{t} d x \\
& -\delta(m+n) u^{m+n-1} u_{t}, \\
\triangle J & =\triangle\left(u_{t}-\delta u^{m+n}\right) \\
& =\triangle u_{t}-\delta(m+n) u^{m+n-1} u_{t}+\delta(m+n) u^{2 m+n-1} \int_{\Omega} u^{n} d y \\
& -\delta(m+n)(m+n-1) u^{m+n-2}|\nabla u|^{2},
\end{aligned}
$$

and

$$
\begin{aligned}
J_{t} & -\triangle J-J m u^{m-1} \int_{\Omega} u^{n} d y-n u^{m} \int_{\Omega} u^{n-1} J d y \\
& \geq n \delta u^{m} \int_{\Omega} u^{2 n+m-1} d y-\delta n u^{2 m+n-1} \int_{\Omega} u^{n} d y \\
& =n \delta u^{m}\left(\int_{\Omega} u^{2 n+m-1} d y-u^{m+n-1} \int_{\Omega} u^{n} d y\right) .
\end{aligned}
$$

Since $m+n>1$ and $n /(2 n+m-1)+(m+n-1) /(2 n+m-1)=1$, 
by Young's inequality, we get

$$
\begin{aligned}
u^{m+n-1} & \left(\int_{\Omega} u^{2 n+m-1} d y\right)^{n /(2 n+m-1)} \\
\leq & \frac{m+n-1}{2 n+m-1}\left(u^{m+n-1}\right)^{(2 n+m-1) /(m+n-1)} \\
& +\frac{n}{2 n+m-1} \int_{\Omega} u^{2 n+m-1} d y .
\end{aligned}
$$

By Hölder's inequality, we have

$$
\int_{\Omega} u^{n} d y \leq\left(\int_{\Omega} u^{2 n+m-1} d x\right)^{n /(2 n+m-1}
$$

Combining (4.2) and (4.3) with (4.1), we obtain

$$
\begin{aligned}
& J_{t}-\triangle J-J m u^{m-1} \int_{\Omega} u^{n} d y-n u^{m} \int_{\Omega} u^{n-1} J d y \\
& \geq n \delta u^{m}\left(\int_{\Omega} u^{2 n+m-1} d y-u^{m+n-1}\right)\left(\int_{\Omega} u^{2 n+m-1} d y\right)^{n /(2 n+m-1)} \\
& \geq n \delta u^{m}\left(\int_{\Omega} u^{2 n+m-1} d y-\frac{m+n-1}{2 n+m-1} u^{m+2 n-1}\right. \\
& \left.-\frac{n}{2 n+m-1} \int_{\Omega} u^{2 n+m-1} d y\right) \\
& =n \delta u^{m} \frac{m+n-1}{2 n+m-1}\left(\int_{\Omega} u^{2 n+m-1} d y-u^{2 n+m-1}\right) \geq 0 .
\end{aligned}
$$

On the other hand, for $(x, t) \in \partial \Omega \times(0, T)$, we have

$$
\begin{gathered}
J=u_{t}-\delta u^{m+n}=\int_{\Omega} l f(x, y) u^{l-1} u_{t} d y-\delta\left(\int_{\Omega} f(x, y) u^{l} d y\right)^{m+n} \\
=\int_{\Omega} l f(x, y) u^{l-1} J d y \\
+\int_{\Omega} l \delta f(x, y) u^{m+n+l-1} d y-\delta\left(\int_{\Omega} f(x, y) u^{l} d y\right)^{m+n}
\end{gathered}
$$


By Jensen's inequality, we know that

$$
\begin{aligned}
J & \geq \int_{\Omega} l f(x, y) u^{l-1} J d y+l \delta\left(\int_{\Omega} f(x, y) u^{l} d y\right)^{(m+n+l-1) / l} \\
& -\delta\left(\int_{\Omega} f(x, y) u^{l} d y\right)^{m+n} .
\end{aligned}
$$

We can choose a suitable constant $l$ satisfying that

$$
l\left(\int_{\Omega} f(x, y) u^{l} d y\right)^{(m+n+l-1) / l} \geq\left(\int_{\Omega} f(x, y) u^{l} d y\right)^{m+n} .
$$

Hence, for such a constant $l$ we get $J \geq \int_{\Omega} l f(x, y) u^{l-1} J d y$. Moreover,

$$
J(x, t)=u_{t}(x, t)-\delta u^{m+n}(x, t)=\triangle u-u^{m} \int_{\Omega} u^{n} d y-\delta u^{m+n},
$$

and so $J(x, 0) \geq 0$, since conditions (C1)-(C2) hold. Therefore, $J(x, t)$ $\geq 0$ for all $(x, t) \in \bar{\Omega} \times[0, T)$, which means that $u_{t} \geq \delta u^{m+n}$ in $\bar{\Omega} \times[0, T)$. Integrating this inequality over $(t, T)$, we get $u \leq C(T-t)^{-1 /(m+n-1)}$, where $C=[\delta(m+n-1)]^{-1 /(m+n-1)}$. Combining this inequality with Lemma 4.1, the desired result follows.

4.2. Blow-up profile. In this section, we assume that $m<1$ and $u(x, t)$ is a blow-up solution of problems (1.1)-(1.3) in finite time. Let $u \sim v$ denote $\lim _{t \rightarrow T} u(t) / v(t)=1$.

Remark 4.2. Using Schauder's fixed point theorem, one can show that the regularized problems of (1.1)-(1.3) admit a unique classical solution $u_{\varepsilon}(x, t)$ on $Q_{T_{\varepsilon}}$ for $p=2$, see $[\mathbf{1 3}, \mathbf{2 6}]$.

Lemma 4.3. Suppose that $\int_{\Omega} \varphi(x, y) d y \geq 1$ for $x \in \partial \Omega, l \geq 1, m<1$ and $m+n>1$. If $\triangle u_{0} \leq 0$ in $\bar{\Omega}$, then $\triangle u \leq 0$ in any compact subset of $\Omega$.

Proof. Let $u_{\varepsilon}(x, t)$ be a solution to problems (1.1)-(1.3) in $Q_{T_{\varepsilon}}$, and let $W=\triangle u_{\varepsilon}$. We then have

$$
W_{t}=\triangle W+m(m-1) u_{\varepsilon}^{m-2}|\nabla u|^{2} \int_{\Omega} u_{\varepsilon}^{n} d y+m u_{\varepsilon}^{m} \triangle u_{\varepsilon} \int_{\Omega} u_{\varepsilon}^{n} d y .
$$

It follows from $u_{\varepsilon}(x, t)>0$ and $m<1$ that $W_{t}-\triangle W \leq m u_{\varepsilon}^{m-1} W \int_{\Omega} u_{\varepsilon}^{n} d y$. 
For $(x, t) \in \partial \Omega \times(0, T)$, we get

$$
\begin{aligned}
W= & u_{\varepsilon}=u_{\varepsilon t}-u_{\varepsilon}^{m} \int_{\Omega} u_{\varepsilon}^{n} d y \\
= & \int_{\Omega} \varphi(x, y) l u_{\varepsilon}^{l-1} u_{\varepsilon t} d y-u_{\varepsilon}^{m} \int_{\Omega} u_{\varepsilon}^{n} d y \\
= & \int_{\Omega} \varphi(x, y) l u_{\varepsilon}^{l-1} W d y \\
& +\int_{\Omega} u_{\varepsilon}^{n} d y\left(l \int_{\Omega} \varphi(x, y) u_{\varepsilon}^{m+l-1} d y-u_{\varepsilon}^{m}\right) \\
\leq & \int_{\Omega} \varphi(x, y) l u_{\varepsilon}^{l-1} W d y \\
& +\int_{\Omega} u_{\varepsilon}^{n} d y\left(l C_{1}^{m+l-1} \int_{\Omega} \varphi(x, y) d y-C_{2}^{m}\right) \\
\leq & \int_{\Omega} \varphi(x, y) l u_{\varepsilon}^{l-1} W d y,
\end{aligned}
$$

where $C_{1}=\max _{x \in Q_{T_{\varepsilon}}} u_{\varepsilon}, C_{2}=\min _{x \in Q_{T_{\varepsilon}}} u_{\varepsilon}$ and $W(x, 0)=\triangle u_{0} \leq 0$, which completes the proof.

Let $g(t)=\int_{\Omega} u^{n} d x$, and let $G(t)=\int_{0}^{t} g(s) d s$.

Lemma 4.4. Under the same conditions as Lemma 4.3, we have

$$
\lim _{t \rightarrow T} g(t)=\lim _{t \rightarrow T} G(t)=+\infty .
$$

Proof. By Lemma 4.3, we know that $u_{t} \leq u^{m} g(t)$. Integrating this inequality over $(0, t)$, we get

$$
\frac{1}{1-m} u^{1-m}(x, t) \leq \int_{0}^{t} g(s) d s+\frac{1}{1-m} u^{1-m}(x, 0),
$$

from which the desired result follows, since $\lim _{t \rightarrow T}\|u\|=+\infty$ and $m<1$.

From now on, we assume that $C$ is a generic positive constant not necessarily the same at different occurrences. 
Lemma 4.5. Under the same conditions as Lemma 4.3, we have

$$
\lim _{t \rightarrow T} \frac{\int_{0}^{t} G^{(1-m) /(1-m-n)}(s) d s}{G(t)}=0 .
$$

Proof. From Theorem 1.10, we know that

$$
\int_{0}^{t} G^{(1-m) /(1-m-n)} d s \leq C \int_{0}^{t}(T-t)^{1-m} d s .
$$

Combining this inequality with (4.4), we obtain

$$
G(t) \geq C u^{1-m-n} \geq C(T-t)
$$

from which the result follows.

Lemma 4.6. Under the same conditions as Lemma 4.3, we have

$$
\lim _{t \rightarrow T} \frac{u^{1-m}(x, t)}{(1-m) G(t)}=\lim _{t \rightarrow T} \frac{\|u(\cdot, t)\|_{\infty}^{1-m}(x, t)}{(1-m) G(t)}=1
$$

for any compact subset of $\Omega$.

Proof. It is obvious that $\limsup _{t \rightarrow T}\left(u^{1-m}\right) /[(1-m) G(t)] \leq 1$ from Lemma 4 , and so we only need to show the opposite inequality.

Let $z(x, t)=G(t)-\left[u^{1-m}(x, t)\right] /(1-m)$, and let $\eta(t)=\int_{\Omega} z(y, t) \phi(y)$ $d y$. If $\phi(x)$ solves the following problem:

$$
\begin{aligned}
-\triangle \phi(x) & =\lambda \phi(x, t), \quad x \in \Omega, \\
\phi(x) & =0, \quad x \in \partial \Omega,
\end{aligned}
$$


and $\int_{\Omega} \phi(x) d x=1$, we have

$$
\begin{aligned}
\eta^{\prime}(t) & =\int_{\Omega}\left(g(t)-u^{-m}(y, t) u_{t}(y, t)\right) \phi(y) d y \\
& =-\int_{\Omega} u^{-m}(y, t) \triangle u(y, t) \phi(y) d y \\
& \leq \lambda \int_{\Omega} u^{1-m}(y, t) \phi(y) d y \\
& \leq \int_{\Omega}(G(t)-z(y, t)) \phi(y) d y \\
& \leq C\left(G(t)+\int_{\Omega} z^{-}(y, t) \phi(y) d y\right),
\end{aligned}
$$

where $z^{-}=\max \{-z, 0\}$. It follows from (4.4) that $\inf _{\Omega} z(x, t) \geq-C$. Then $z^{-} \leq C$ and

$$
\eta^{\prime}(t) \leq C G(t)+C^{\prime}
$$

Integrating the inequality above over $(0, t)$, we get

$$
\eta(t) \leq C\left(1+\int_{0}^{t} G(s) d s\right)
$$

which implies that

$$
\int_{\Omega}|z(y, t)| \phi(y) d y \leq C\left(1+\int_{0}^{t} G(s) d s\right) .
$$

Setting $K_{\rho}=\{y \in \Omega \mid \operatorname{dist}(y, \partial \Omega)>\rho\}$, we get

$$
\sup _{K_{\rho}} z(x, t) \leq \frac{C}{\rho^{N+1}} C\left(1+\int_{0}^{t} G(s) d s\right),
$$

since $-\triangle z \leq 0$ in $\Omega \times(0, T)$. By inequality (4.4), we obtain

$$
-\frac{C}{G(t)} \leq 1-\frac{u^{1-m}}{(1-m) G(t)} \leq \frac{C}{\rho^{N+1}} \frac{C\left(1+\int_{0}^{t} G(s) d s\right)}{G(t)},
$$

from which and from Lemmas 4.4 and 4.5 the desired result follows.

Remark 4.7. Lemma 4.6 implies that the blow-up set is the whole domain $\Omega$ if the conditions of Lemma 4.3 are satisfied. 
Proof of Theorem 1.11. By Lemma 4.6, we have

$$
G^{\prime}(t)=\int_{\Omega} u^{n} d x \sim|\Omega|[(1-m) G(t)]^{m /(1-m)} \quad \text { as } t \rightarrow T .
$$

Moreover, we can obtain

$$
\begin{gathered}
G(t) \sim(1-m)^{-1}\left(\left(|\Omega| \frac{m+n-1}{1-m}\right)^{-1 /(m+n-1)}\right. \\
\left.(1-m)^{-1 /(m+n-1)}\right)^{1-m}(T-t)^{(m-1) /(m+n-1)} \\
=(1-m)^{-1} M_{1}^{1-m}(T-t)^{(m-1) /(m+n-1)}
\end{gathered}
$$

where

$$
M_{1}=\left(|\Omega| \frac{m+n-1}{1-m}\right)^{-1 /(m+n-1)}(1-m)^{-1 /(m+n-1)} .
$$

This completes the proof.

Acknowledgments. The authors would like to express their sincere gratitude to the anonymous reviewers for their insightful and constructive comments.

\section{REFERENCES}

1. W. Allegretto, G. Fragnelli, P. Nistri and D. Papin, Coexistence and optimal control problems for a degenerate predator-prey model, J. Math. Anal. Appl. 378 (2011), 528-540.

2. J. Bebernes and D. Eberly, Mathematical problems from combustion theory, Springer-Verlag, New York, 1989.

3. A. Calsina, C. Perello and J. Saldana, Non-local reaction-diffusion equations modelling predator-prey coevolution, Publ. Mat. 32 (1994), 315-325.

4. Z.J. Cui and Z.D. Yang, Roles of weight functions to a nonlinear porous medium equation with nonlocal source and nonlocal boundary condition, J. Math. Anal. Appl. 342 (2008), 559-570.

5. W.A. Day, Extensions of property of heat equation to linear thermoelasticity and other theories, Quart. App1. Math. 40 (1982), 319-330.

6. A decreasing property of solutions of parabolic equations with applications to thermoelasticity, Quart. App1. Math. 40 (1983), 468-475.

7. K. Deng and H.A. Levine, The role of critical exponents in blow-up theorems: The sequel, J. Math. Anal. Appl. 243 (2000), 85-126. 
8. J.I. Diaz, Nonlinear partial differential equations and free boundaries, I. Elliptic problems, Res. Notes Math. 106, Pitman, London, 1985.

9. A. Friedman, Monotonic decay of solutions of parabolic equations with nonlocal boundary conditions, Quart. Appl. Math. 44 (1986), 401-407.

10. J. Furter and M. Grinfield, Local vs. non-local interactions in populations dynamics, J. Math. Biol. 27 (1989), 65-80.

11. A.L. Gladkov and K.L. Kim, Blow-up of solutions for semilinear heat equation with nonlinear nonlocal boundary condition, J. Math. Appl. 338 (2008), 264273.

12. F.C. Li and C.H. Xie, Global and blow-up solutions to a p-laplace equation with nonlocal source, Comput. Math. Appl. 46 (2003), 1525-1533.

13. Global existence and blow-up for a nonlinear porous medium equation, Appl. Math. Lett. 16 (2003), 185-192.

14. Z.G. Lin and Y.R. Liu, Uniform blow-up profiles for diffusion equations with nonlocal source and nonlocal boundary, Acta. Math. Sci. 24 (2004), 443-450.

15. D.M. Liu and C.L. Mu, Blow-up properties for a semilinear reaction-diffusion system with nonlinear nonlocal boundary conditions, Abstr. Appl. Anal. 2010 (2010), 17 pages, Article ID 148035, doi:10.1155/2010/148035.

16. C.P. Niculescu and L. Roventa, Generalized convexity and the existence of finite time blow-up solutions for an evolutionary problem, Nonl. Anal. 75 (2012), $270-277$.

17. C.V. Pao, Nonlinear parabolic and elliptic equations, Plenum Press, New York, 1992.

18. _ Asymptotic behavior of solutions of reaction-diffusion equations with nonlocal boundary conditions, J. Comput. Appl. Math. 88 (1998), 225-238.

19. - Numerical solutions of reaction-diffusion equations with nonlocal boundary conditions, J. Comput. Appl. Math. 136 (2001), 227-243.

20. S. Seo, Global existence and decreasing property of boundary values of solutions to parabolic equations with nonlocal boundary conditions, Pacific J. Math. 193 (2000), 219-226.

21. P. Souplet, Blow-up in nonlocal reaction-diffusion equations, SIAM J. Math. Anal. 29 (1998), 1301-1334.

22. Y.L. Wang, C.L. Mu and Z.Y. Xiang, Blow-up of solutions to a porous medium equation with nonlocal boundary condition, Appl. Math. Comp. 192 (2007), $579-585$.

23. Properties of positive solution for nonlocal reaction diffusion equation with nonlocal boundary, Bound. Value Probl. 2007 (2007), 12 pages, Article ID 64579, doi:10.1155/2007/64579.

24. Y.F. Yin, On nonlinear parabolic equations with nonlocal boundary condition, J. Math. Anal. Appl. 185 (1994), 54-60.

25. S.N. Zheng and L.H. Kong, Roles of weight functions in a nonlinear nonlocal parabolic system, Nonl. Anal. 68 (2008), 2406-2416. 
26. S.N. Zheng and L.D. Wang, Blow-up rate and profile for a degenerate parabolic system coupled via nonlocal sources, Comp. Math. Appl. 52 (2006), 13871402.

27. S.L. Zhou, A priori $L^{\infty}$ estimate and existence of solutions for some nonlinear parabolic equations, Nonl. Anal. 42 (2000), 887-904.

School of Mathematical Sciences, Ocean University of China, Qingdao 266100, P.R. ChinA

Email address: fangzb7777@hotmail.com

School of Mathematical Sciences, Ocean University of China, Qingdao 266100, P.R. China

Email address: jianyun.6@163.com 